comprehensive feeling for the subject. In places, the student is confronted with partial derivatives, vectors and laplacians. $\mathrm{He}$ will not be familiar with such notation, and, although its meaning is explained as it is introduced, some difficulty must be anticipated. (For this reason the misprint in the first use of vector notation on page 91 is regrettable.) Mathematical complexity reaches its height in the account of computer-based forecasting where key new ideas are introduced almost line by line with baffling brevity. No links are actually missing, however, and the tenacious will be rewarded. Let reluctant mathematicians be undeterred by that warning; they will find much which is interesting and succinctly explained. Some chapters, for example those on rain processes and on methods of observation, require no mathematics at all, and others require very little. Physics teachers may extract from these a fund of illustrations, useful to the lower school.

The style is rather disjointed and I was distracted by the number of footnotes and references to sections later in the book, but the attractive finish, the wealth and authority of the information and the reasonable price more than compensate.

R. S. HARWOOD

\section{SOUTHERN AFRICAN GEOLOGY}

\section{Geological History of Southern Africa}

By S. H. Haughton. Pp. $535+16$ plates. (The Geological Society of South Africa: Johannesburg, 1969.) R10.50.

Southern AFRICA is of exceptional geological interest, as well as of immense economic importance on account of its mineral resources. For an account of the geology of this region one formerly referred to the Geology of South Africa by A. L. du Toit, which is one of the best books on regional geology ever written. Dr Haughton revised the third edition, which was published in 1954 , but, since then, so much new work has been carried out that a completely new book became necessary. Nobody is better qualified to evaluate the formidable mass of geological data relating to Southern Africa than Haughton, who has been in the forefront of South African geology for more than half a century. He has written a careful, judicially balanced and authoritative account, which will serve as a standard reference on the geology of southern Africa for many years.

One inevitably compares the new book with du Toit's masterpiece. Haughton is less successful. His arrangement, partly chronological, partly geographical, is confusing. Regional correlation tables, with ages, would have helped the reader, and more maps and diagrams are needed (some of those used are poor). Too much attention is paid to stratigraphic nomenclature and too little to fundamental problems. One looks in a new book on the geology of southern Africa for an up to date account of some of the geological wonders of that region. There are the very old rocks of the Barberton mountainland which are being taken as a model for the earliest stages in the formation of continental crust; the great dyke of Rhodesia and the Bushveld complex of the Transvaal are two enormous bodies of basic igneous rock in which the separation of layers of erystals has produced remarkable mineral concentrations. Around Kimberley and elsewhere there are pipes which contain not only diamonds but also samples carried up from the upper mantle. The Vredefort dome, surrounded by a radial array of shatter cones, has been interpreted as a huge meteorite impact structure. There are fascinating problems connected with the concentration of gold and uranium in the Witwatersrand conglomerate beds. The Dwyka glaciation of southern Africa in the Carboniferous period was for long the most important evidence for continental drift. The Karroo vertebrates on which Haughton is an authority, and the australopithecines from the cave deposits in the
Transvaal, are of outstanding interest. Haughton, in this book, does not succeed in leading the reader to appreciate the interest and significance of these and other such phenomena, nor does he provide a clear view of the present state of knowledge regarding them. Much of this new knowledge depends on geophysical and to some extent geochemical work to which Haughton pays too little attention. For example, the palaeomagnetic evidence which tells us about the latitude and orientation of the southern African continent through geological time is almost ignored. Radiometric age determinations are treated rather as though they were an innovation of still doubtful value.

The coloured geological map of southern Africa on a scale of $1: 5,000,000$ is much clearer than the corresponding map in du Toit's book, besides containing a great deal of new detail. The price of the book is low; it is a good buy.

R. M. SHACKLETON

\section{PLASMA THEORY AND OBSERVATION}

Plasma Waves in Space and in the Laboratory

Vol. 1. Edited by J. O. Thomas and B. J. Landmark. (Proceedings of NATO Advanced Study Institute, Røros, Norway, 17-26 April 1968.) Pp. 487. (Edinburgh University Press: Edinburgh, September 1969.) 160s.

THxs volume, the first of two reporting on the Røros school, is composed of more than twenty papers, of a basic introductory or review nature (rather than current research, scheduled for volume two). The topics range widely to cover theory of probes, observations in the laboratory, in the ionosphere and in interplanetary space and theory of plasma resonances and VLF emissions-that is, "space" plasmas in the local, accessible and non-astrophysical sense. In a short review, it is impossible to comment on the papers individually: however, common to many of the contributions is an emphasis on squaring theory with observation. This is meeting with increasing success as is particularly evident in Crawford's discussion of wave propagation in stable laboratory plasmas, and in studies with ionospheric probes (Balmain and Otsiutik). The importance, for the advancement of plasma science, of space plasma as an experimental medium is brought up in a very readable introductory paper on ionospheric resonances (Thomas and Andrews) - free from boundaries, the plasma has relative uniform density, is fully ionized and virtually collisionless. With the development of resonance techniques and probe systems (cf. Storcy, Aubry and Meyer on their quadripole probe), sophisticated experiments on natural plasmas are becoming a reality.

The blurb on the dust-jacket claims a role as an up to date textbook, but apart from very few sections-notably the illuminating "Rowlands" theory of plasma wavesthe book reports completed work rather than useful approaches. Problems of non-linear waves and turbulence are hardly touched on. Moreover, in trying to be a textbook in excluding both abstracts and conference discussion, the book loses potential usefulness. Comments on the significance and validity of a piece of work-that important and unique contribution of a conferenceare lost.

On the other hand, I would congratulate the editors and publishers in producing an attractive, high quality book. There are few printing errors, except in the paper on pitchangle instabilities, where misprints and poor format are annoying. An error of more substance occurs in Frederick and Scarf's paper on cyclotron modes, where the substitution of $\beta_{+}$for $\beta_{-}$in equation (14) ignores one region of validity of the classical Bernstein modes. Plasma physicists of diverse interests will indeed find this volume both interesting and useful in cross-fertilizing our fast-diverging studies of plasmas, but its importance as a student text can be only minor.
M. K. Wallis 